\title{
¿Pueblo feminista? Algunas reflexiones en torno al devenir popular de los feminismos
}

\section{Feminist people? Some considerations on the popular development of feminisms}

\author{
Natalia Martínez*
}

\begin{abstract}
RESUMEN: En este artículo se analiza y discute desde los presupuestos teóricos de algunos estudios que sostienen la emergencia de un pueblo feminista, así como los que cuestionan las interpretaciones del populismo como única vía de construcción de un pueblo. Se exponen, asimismo, las movilizaciones de mujeres y feministas devenidas del \# NiUnaMenos, y se concluye con una propuesta de análisis centrada en la práctica políitica del rechazo y sus efectos políiticos de subjetivación.
\end{abstract}

Palabras clave: Pueblo, Populismo, Feminismos.9

ABSTRACT: The present paper analyses and discusses theoretical assumptions of some studies that argue the emergence of a feminist people, as well as those that question the interpretations of populism as the only way to build a people. Making up one of the first interpretations of the mobilizations of women and feminists that rose from \# NiUnaMenos, it ends up in a proposal of analysis centered on the political practice of rejection and its subjectivization political effects.

Key wORDS: People, Populism, Feminisms.

Instituto de Humanidades-CONICET/Área Feminismos Género y Sexualidades, Centro de Investigaciones-Facultad de Filosofía y Humanidades-Universidad Nacional de Córdoba (nataliammp@gmail.com). 


\section{INTRODUCCIÓN $^{1}$}

un cuando la violencia hacia las mujeres ha sido combatida por
los feminismos latinoamericanos, anual y públicamente, cada
25 de noviembre desde 1981, la demanda se hizo masiva sólo
hace un par de años, tras la emergencia de un desgarrador grito: "iNi una menos!", difundido a través de las redes sociales. El grito irrumpió por la fuerza del horroroso crimen de Chiara Páez, ${ }^{2}$ aunque claramente excedió ese marco. Tras la primera y masiva convocatoria del 3 de junio del 2015, organizada por una plataforma de comunicadorxs, representantes de la cultura y otros sectores sociales y políticos cercanos y/o activos de los feminismos, el grito iNi una menos! se ha multiplicado y extendido, abrazando otros reclamos y fechas históricas de las feministas y colocándolos, como nunca antes, en el primer plano de las agendas nacionales, de la región y del mundo. Es en este nuevo marco donde las luchas y reivindicaciones feministas se entrelazan y resignifican, desde donde asistimos a la redefinición y fortalecimiento de un día histórico para las luchas de mujeres y feministas, como lo es el 8 de marzo. Rebautizado "8M", como en la actualidad se nombran los días que marcan nuestra historia, el reconocimiento tradicional a las mujeres trabajadoras se proclama, desde el 2016, bajo la modalidad de un "Paro Internacional de Mujeres" (PIM) con un acatamiento en más de cincuenta países. ${ }^{3}$ En este sentido, las repercusiones del \# Num no sólo han impactado el contexto político y social de Argentina, sus efectos se vislumbran - siguiendo la metáfora de las genealogías feministas en el formato de "olas"- como el devenir de una marea feminista internacional.

El presente artículo emerge de algunas interrogantes que se abren gracias a este novedoso y potente proceso de extensión del ideario feminista, en particular, atendiendo a las posibilidades y limitaciones de con-

1 El presente trabajo forma parte de una investigación en curso, titulada: "Feminismos Populares: entre la política de la calle y la política estatal", financiada por el CONICET.

2 Chiara Pérez era una adolescente embarazada de 14 años, quien fue asesinada por su novio de 16. El crimen fue encubierto por su familia. Véase la nota de Lorena Panzerini 2017.

3 Véase el sitio: parodemujeres.com 
cebir un "pueblo feminista", bajo ciertas consideraciones generales que se efectuarán desde el caso de Argentina. Con ese propósito, el recorrido propuesto se inicia con la reflexión sobre el precursor análisis de Graciela Di Marco, quien avizoró un proceso hegemónico del feminismo popular a partir de la Campaña por el derecho al aborto legal, seguro y gratuito, en el marco de los procesos de movilización y organización social acaecidos después de la crisis del 2001. Considero que la investigación de Di Marco es provocadora y sugerente no sólo por haber examinado la vinculación del feminismo con las movilizaciones populares -abordaje que suele ser muy limitado entre los estudios feministas- sino por comprender esa vinculación como una articulación populista, constitutiva de un pueblo feminista, en los términos de la obra de Ernesto Laclau. Con el fin de examinar las posibilidades de extender esta lectura a los procesos actuales devenidos por el \#NiUnaMenos (NUM), en un primer apartado me detengo en lo que considero son algunos de los presupuestos que sostuvieron a la legalización del aborto como una "demanda popular" en la lectura de Di Marco, en particular, aquellos que anclan la comprensión de "lo popular" a criterios sociodemográficos, así como "el" feminismo a una perspectiva epistemológica privilegiada en la definición de los "intereses estratégicos de género". En un segundo apartado completo esta tarea, específicamente a partir de las tres precondiciones que Laclau hiciera sobre la emergencia de una identidad populista. Advirtiendo las posibilidades y limitaciones de una primera lectura respecto de las movilizaciones feministas en estos términos, termino el análisis con un tercer enfoque inspirado en la obra de Jacques Rancière.

\section{¿PUEBLO FEMINISTA?}

Graciela Di Marco (2011) es una de las pioneras en hacer referencia al "pueblo feminista" a partir de un análisis de las movilizaciones de mujeres y feministas desencadenadas tras los sucesos políticos y sociales del 2001 en Argentina. En el marco de la teoría de Ernesto Laclau, la autora anali- 
za la inclusión de algunas demandas feministas en los debates internos de las asambleas post-2001 -en el movimiento piquetero y en empresas recuperadas - como un proceso de construcción de un "pueblo feminista" o, en otras palabras, como un proceso hegemónico de un feminismo popular. A diferencia de lo que venía sucediendo en los noventa, periodo en el que desde diversos frentes se procuraba instalar la oposición entre feminismo y crisis social, "con el argumento de que el debate por los intereses estratégicos de género postergaba o restaba importancia a los grandes problemas sociales del país" (Di Marco 2011: 270), la autora entiende que el contexto post-2001, en particular desde el Encuentro Nacional de Mujeres (ENM) de Salta en 2002, hizo visible la participación de piqueteras, asambleístas, sindicalistas, como nuevas expresiones del activismo de mujeres que comenzaban a reconocerse desde los feminismos. ${ }^{4}$ El feminismo popular emerge de este nuevo contexto de la mano de la articulación entre feministas y mujeres de los sectores populares a partir de los "tres derechos fundamentales" que, para Di Marco, caracterizan sus demandas: el trabajo digno, la lucha en contra de la violencia hacia las mujeres, y la legalización del aborto (Di Marco 2011: 295). Desde su perspectiva, la extensión del ideario feminista entre las "mujeres populares" se habría producido en torno a la demanda por la legalización del aborto, como una "demanda popular", en términos de Laclau. Es decir, a diferencia de las demandas por trabajo digno y en contra de la violencia hacia las mujeres, que serían "demandas democráticas" - esto es, que pueden ser atendidas por el sistema institucional del Estado y, de esa manera, ser desactivadas como un motivo de lucha colectiva- la reivindicación por la legalización del aborto encarnaría un "significante de la ciudadanía plena de las mujeres, del laicismo y el pluralismo", una demanda que excede su particularidad y puede sostenerse como un proyecto político antagonista al seguido por la jerarquía de la Iglesia católica (Di Marco 2011: 296). Este proyecto habría comenzado a emerger en el ENM del 2003 en Rosario

\footnotetext{
Di Marco cita, en este sentido, una de las consignas que Liliana Daunes recuperaba de aquel periodo: "Vamos a hacer la patria socialista, la vamos a hacer piquetera y feminista" (Di Marco 2011: 270).
} 
cuando, desde su perspectiva, se produce un punto de inflexión: tanto por la presencia de sectores vinculados a la Iglesia católica, resueltos a impedir la discusión en torno al aborto, como de los partidos políticos de izquierda, que pusieron todo el énfasis en las luchas sociales devenidas de la crisis económica, sin vincularlas a las luchas de las mujeres. Además, según Di Marco, ése fue un año decisivo porque en ese ens "el feminismo, que en anteriores luchas había basado su accionar en la alianza con las mujeres políticas [...], se articulaba ahora con las mujeres populares" (Di Marco 2011: 289). Esta situación sería la que habría posibilitado la radicalización de las demandas feministas y la emergencia de un pueblo feminista en torno a la demanda por la legalización del aborto, como un significante vacío que "condensa la lucha por su ciudadanía, esto es, ejercer la libertad sobre sus propios cuerpos” (Di Marco 2011: 302).

Atendiendo a los procesos que se sucedieron desde que Di Marco escribiera su libro, en especial las movilizaciones del \#Ni una menos y el reciente "8M", considero que es preciso retomar su análisis y preguntarnos por las condiciones actuales de ese pueblo feminista. Como un primer elemento a examinar, propongo la reconsideración de la demanda por el derecho al aborto como una demanda popular tal y como fuera definida por Ernesto Laclau (2005). Recordemos que, para el autor, las demandas populares son las que habilitan la constitución de una subjetividad social más amplia, que excede a la propia demanda, a través de una articulación equivalencial entre demandas (Laclau 2005: 99). En otras palabras, una demanda se hace popular cuando se universaliza y, de ese modo, ya no sólo representa el sentido particular que la identificaba, sino otras nuevas que se van encadenando al sentido cada vez más amplio que se inscribe en su nombre. Esta condición no se encuentra en la propia demanda, porque no existe una demanda con un "destino manifiesto", 5 sino que es un proceso que se verifica en el propio devenir de la demanda, en los procesos de articulación singulares que se ven posibilitados por su pregnancia, por la contaminación y sobredeterminación de sentidos que su circulación va

5 'Ninguna demanda social tiene como su 'destino manifiesto' una forma a priori de inscripción -todo depende de una lucha hegemónica-" (Laclau 2009: 63). 
habilitando. Si retomamos el análisis de Di Marco, es cierto que la Campaña por el derecho al aborto legal, seguro y gratuito sigue creciendo y hoy moviliza a diversos sectores sociales históricamente renuentes a hacerlo. La campaña ha presentado por séptima vez un proyecto de ley al Congreso argentino -que en los días que corren ya cuenta con media sanción en la Cámara de Diputadxs-, en un marco de creciente apoyo, logrando instalar la demanda como una de las grandes deudas de la democracia. Pero, al mismo tiempo, es poco plausible considerar que es una demanda que se ha universalizado; más bien al contrario, sigue siendo una de las demandas más costosas, en términos políticos, de los feminismos. ${ }^{6}$ Tan costosa que no pudo ni erigirse como demanda en la primera movilización del Num, fundamentalmente promovida por los feminismos. ${ }^{7}$ En este marco, la posibilidad de que la demanda por el derecho al aborto fuese considerada en el 2011 como una demanda popular fue quizás el resultado, no el que Di Marco lo haya verificado en su proceso de articulación, sino más bien de un presupuesto. El establecimiento de las demandas de "las mujeres populares" - como trabajo digno, contra la violencia y el derecho al aborto-y su interpretación distintiva como "demandas democráticas" (las dos primeras) o "demandas populares" (la última) parece sostenerse sobre una lectura previa en términos de intereses "prácticos" y "estratégicos”, según la reconocida definición de Maxine Molineaux (1985). Esto

6 Véanse los comunicados de la Iglesia católica: https://www.pagina12.com.ar/121593nos-duele y de algunas instituciones hospitalarias: https://www.clarin.com/sociedad/ aborto-crece-presion-permita-objecion-conciencia-institucional_0_HJhg_rdbX.html Véase Ni Una Menos, sitio web oficial.

8 En uno de sus primeros trabajos, Molineaux estableció una distinción conceptual muy influyente entre los "intereses de las mujeres" y los "intereses de género". Para Molineaux, los intereses de las mujeres son múltiples y variados porque están posicionadas socialmente de forma diferencial según su clase, etnia o nacionalidad y ello deriva, desde su perspectiva, en la imposibilidad de hacer generalizaciones sobre sus intereses. Es decir, para ella se trata de una imposibilidad epistemológica, no política. Molineaux no aprecia la politicidad inherente a la definición de los intereses, incluso cuando advierte que de la imposibilidad epistemológica para hacerlo se deriva una consecuencia eminentemente política: los intereses de género no son, necesariamente, los intereses "primarios" de las mujeres (Molineau 1985: 231). Atendiendo a esa consecuencia y admitiendo que el sexo no proporciona una "suficiente base para 
es, para Di Marco el derecho al aborto se erige como demanda popular en tanto siempre fue interés estratégico para las feministas. No así las demandas por el trabajo digno y la lucha en contra de las violencias hacia las mujeres, que suelen ser sostenidas por los sectores populares y, de este modo, usualmente leídas como intereses prácticos.

\section{"Mujeres" y "feministas" \\ o sobre la politicidad de las fronteras}

Incluso reconociendo ciertos contornos de la distinción entre el "movimiento de mujeres" y "las feministas" como una de las primeras diferencias del activismo, ${ }^{9}$ ésta no llega a establecerse explícitamente hasta

asumir intereses comunes", opta por realizar una distinción entre los "intereses de las mujeres" y los "intereses de género". Los primeros, para identificar los intereses que las mujeres reclaman "como propios", aunque no se identifiquen "con cuestiones de género" (1985: 232). Los segundos, como aquellos intereses que "se desarrollan en virtud de la posición social derivada de los atributos de género" (1985: 232). Y sin llegar a definir cuáles son esas "cuestiones de género" ni sus "atributos", realiza una segunda distinción en el interior de los intereses de género, entre "intereses prácticos" e "intereses estratégicos", como una de las distinciones más polémicas y difundidas entre los estudios de Género en el Desarrollo (GED) (Guzmán, Portocarrero, Vargas 1991; Moser 1995). Los intereses prácticos son intereses inductivos que se basan en las necesidades y condiciones concretas de las mujeres que surgen de su emplazamiento dentro de la división sexual del trabajo. Son intereses que se formulan inmediatamente desde ese posicionamiento por las mismas mujeres, sin "intervenciones externas" (Molineaux 1985: 233). Los estratégicos, en cambio, son derivados de forma deductiva y aspiran a "transformar las relaciones sociales con el fin de potenciar la posición de las mujeres y conseguir un reposicionamiento más duradero dentro del ordenamiento de género y la sociedad en general" (Molineaux, 1985: 232). Son los intereses mediados por una concepción feminista o, en palabras de la autora, los que se considerarían como "verdaderos intereses" para las feministas (Molineaux 1985: 233). Y aunque esta definición no establece explícitamente que los intereses estratégicos sean sostenidos exclusivamente por las feministas, y los prácticos por el resto de las organizaciones de mujeres, ésta es la lectura predominante que se produjo en los estudios sobre la movilización y organización femeninas-feministas en la década de los ochenta, como es el caso de los análisis de Elizabeth Jelin $(1987,1994)$.

9 Me refiero a las distinciones que fueron habilitando la configuración identitaria de "las feministas" en relación con otras movilizaciones y activismos "de mujeres no-fe- 
comienzos de la década de los ochenta, con la masiva participación de las mujeres en los procesos abiertos por las transiciones democráticas de la región. Una de las referencias más citadas para establecer esa distinción en aquel periodo es el trabajo anteriormente referenciado de Maxine Molineaux. Aun cuando el enfoque propuesto por Molineaux aspiraba a una comprensión de los "intereses femeninos como histórica y culturalmente constituidos", incluso "como política y discursivamente construidos" (Molineaux 2003: 236), la lectura dominante, también habilitada por la propia autora, ${ }^{10}$ se sostenía en una distinción previa entre las activistas, definidas sociológicamente en términos de clase, de sectores sociales: entre las mujeres cuyas necesidades inmediatas les impiden luchar por "su liberación", por sus "intereses verdaderos" mediados por el feminismo, y las feministas, quienes no tienen ese problema. De lo contrario, ¿cómo se llega a la conclusión de que los intereses devenidos de la división sexual del trabajo son "prácticos"?, ¿y cómo llegan a definirse los intereses de las feministas como "estratégicos"? Para Molineaux, la practicidad de los intereses deviene de su inmediatez, regida por la premura de quien no puede sino intentar resolver las necesidades apremiantes de su vida cotidiana y de su devenir no mediado por una concepción feminista de transformación y emancipación social. Y aunque ella reconozca y asuma las diferencias que implican distintas concepciones políticas a la hora de comprender una práctica como estratégica (reconociendo esas diferencias incluso en

ministas". Porque, quizás valga la pena remarcar, identificarse como "feminista" siempre implicó la institución de fronteras con otros proyectos políticos disponibles, entre otros, el de anarquistas, peronistas, católicas. Otras veces, esas diferencias fueron disputadas hacia el interior de lo que se concebía como "verdadero feminismo". Para un análisis de la emergencia del "feminismo" como categoría identitaria en Argentina, véase Martínez 2015.

10 La frase anteriormente citada proviene de un párrafo que dice: "Volviendo por un momento a mi artículo original [se refiere al artículo de 1985], la intención era problematizar el modo como se formulaban los intereses femeninos, así como la idea de que el sexo proporcionase suficiente base para asumir intereses comunes. Por el contrario, consideraba los intereses femeninos como histórica y culturalmente constituidos, un reflejo del emplazamiento social específico y las prioridades de grupos concretos de mujeres, aunque no reducibles a ellos; también los consideraba como política y discursivamente construidos (Molineaux 2003: 236). Énfasis agregado. 
el interior de los feminismos), afirma que: "en la formulación de intereses prácticos se asume una conformidad con el ordenamiento de género existente, mientras que, en el caso de los intereses estratégicos, se cuestionan explícitamente dicho ordenamiento y la conformidad de algunas mujeres con él" (Molineaux 1998: 242). En otras palabras, quienes se movilizan desde sus intereses prácticos (léase, las mujeres de los sectores populares), lo hacen sin mediaciones políticas y son conformistas ante el orden existente; y quienes lo hacen desde los estratégicos (las feministas), lo hacen habilitadxs por una mediación feminista y, de ese modo, son críticxs de ese orden. Pero, ipor qué no considerar, directamente, que las mujeres de los sectores populares tengan otros intereses estratégicos?, ¿que se movilizan desde otras mediaciones? Su planteamiento presupone un feminismo, en singular, como única mediación crítica y emancipatoria de las mujeres - en términos de comprensión de una realidad objetiva y la provisión de fundamentos autorizados para sus problemas-, cuyo conocimiento las habilita a erigir reclamos legítimos de forma exclusiva.

Sin poder llegar a abordar en este análisis los presupuestos epistemológicos y, agregaría, ontológicos de este tipo de concepciones, tarea que he emprendido en trabajos anteriores (Martínez 2014), ${ }^{11}$ quisiera al menos señalar algunos de sus efectos políticos. El principal es la adjudicación al "feminismo"de un saber privilegiado sobre los intereses de "las mujeres"; y su corolario, que es la desestimación de cualquier otra concepción que pretenda hacerlo. El segundo, que se deriva de lo señalado, es que persiste la referencia "al" feminismo, incluso cuando se habla de "las feministas" y sus diferentes estrategias, como única concepción a la hora de erigir demandas o plantear intereses "de género". Y en este privilegio subyace la idea de que la definición de esos intereses se puede deducir de un orden social dado, como "el patriarcado", sin llegar a atender que el proceso de definición de esos intereses no está exento de interpretaciones divergentes y disputas políticas, algunas irreconciliables. El tercer efecto político de este tipo de concepciones es la insistencia en referirse

11 Para un excelente análisis de estos presupuestos en los debates de los feminismos estadounidenses de la década del ochenta y noventa, véase Linda Zerilli 2008. 
a "las mujeres" como si fuesen un grupo social dado, pre-existente -insistencia que persiste en algunas perspectivas como indispensable a la hora de configurar las demandas de los feminismos, incluso en términos de un esencialismo estratégico. Esta asunción es problemática, no porque "las mujeres" sostengan relaciones sociales, raciales, sexuales y económicas divergentes, sino porque ninguna de esas relaciones implica una definición de intereses de forma inmediata, es decir, que no está mediada por una lectura política de esas relaciones.

Una manera de sortear este tipo de presupuestos, y sus efectos, que la mayoría de las veces se cuela inadvertidamente en los análisis que distinguen entre un "movimiento de mujeres" y "las feministas", es abordando las demandas desde las condiciones singulares de su emergencia, desde los lenguajes disponibles con los que se identifican y las tradiciones políticas que las articulan, sean o no feministas. Hay que advertir cómo juega cada una de esas tradiciones - que pueden ser peronistas, sandinistas, anarquistas, socialistas, entre tantas otras-como lenguajes políticos disímiles a disposición, compitiendo por la articulación de cada una de las demandas y la inscripción de fronteras entre quienes aspiran representar y quienes se dirimen a enfrentar. El reconocimiento de esas tradiciones como un punto de partida, y el carácter eminentemente político (no epistémológico, tampoco sociológico) de la definición de sus fronteras, de sus "intereses estratégicos", es indispensable para comprender los desafíos actuales de una eventual "popularización" de los feminismos.

FEMINISMO POPULAR ¿O POPULISTA?

En el documento leído en la primera movilización del 3 de junio de 2015, el lema que históricamente ha definido a los feminismos, "lo personal es político", parecía ser el principal argumento para combatir la comprensión de los femicidios como un problema de seguridad nacional. Pero ese punto de partida fue ampliamente excedido, incluso unos meses después y en fechas que ni siquiera se ajustan al calendario feminista de la región, 
para incluir demandas cada vez más diversas y heterogéneas al propio legado feminista del país. Como fuera señalado por María Pía López, un 19 de mayo:

[...] el documento leído en la Plaza de Mayo incorporó la cuestión de la criminalización de la protesta, la situación de las mujeres en las cárceles y los distintos modos de explotación. Ni una menos toma contenidos heterogéneos y plantea nuevos problemas. En cada movilización, asamblea, documento, acción en las redes, se fue ampliando un modo de afirmar que Vivas nos queremos. Vivas, no sólo en términos biológicos. Vivas: libres, autónomas, deseantes, productivas. El movimiento de mujeres crece, como un río, creando nuevos cauces y caminos (López, s/f).

En el 2016 el Num, efectivamente, crece e incorpora la demanda por la legalización del aborto. Al año siguiente, se reinscribe en una fecha histórica como el 8 de marzo, llamando a un excepcional Paro Internacional de Mujeres, extendiendo las movilizaciones en su nombre a más de cincuenta países en todo el mundo. En el 2018 esta fecha, devenida en "8M", llegó a movilizar a medio millón de personas en Argentina. De estos procesos surgen demandas que exclaman que "no hay Num sin tierra, trabajo, salud, educación y vivienda". ${ }^{12}$ En este sentido, y como señalaba María Pía López, está claro que el movimiento crece, pero ies un "movimiento de mujeres" lo que crece? Desde el 3 de junio del 2015 a la fecha, el num ha excedido la propia demanda de la que lo vimos emerger. Aun cuando en el primer documento leído ese día, las demandas no se limitaron a la denuncia sobre la violencia ejercida sobre las mujeres, poniendo en evidencia la figura del femicidio, los reclamos que se enmarcan en las movilizaciones convocadas desde el Num son cada vez más amplios y heterogéneos. Como llegaron a señalar en el primer llamamiento al Paro Internacional de Mujeres: "Nos organizamos en todas partes: en las casas, en las calles, en los trabajos, en las escuelas, en las ferias, en los barrios. La fuerza de nuestro movimiento está en los lazos que creamos entre nosotras. "Nos organizamos para

12 Tomado del folleto: "Todos los días construyendo feminismo popular", Confluencia $1^{\circ}$ de Octubre MRT, MTK (Movimiento Tupaj Katari), MPLD (Movimiento Popular La Dignidad), OPSA (Organización Político Social Argentina). 
cambiarlo todo". ${ }^{13}$ ¿Cómo podemos comprender esta creciente amplitud y heterogeneidad propia del Num?, ¿cuáles son las lógicas de articulación que dominan en las movilizaciones y prácticas del Num?

Una manera para comenzar a abordar estas preguntas sería analizando si la misma demanda "Ni Una Menos" se hizo popular, en términos laclaunianos, habilitando que en su nombre se inscriban nuevas demandas, cada vez más amplias y heterogéneas. En esta línea, incluso, podríamos llegar a preguntarnos si la lectura del derecho al aborto como un interés estratégico de los feminismos no impidió que Di Marco reconociera el potencial popular de la demanda en contra de la violencia hacia las mujeres, como "la demanda" que habilitaría la configuración de un pueblo feminista. Pero, ¿es eso lo que está sucediendo?, ¿podemos reconocer una articulación populista en el Num? Señalemos brevemente que la definición de Ernesto Laclau sobre la lógica de articulación populista descansa en tres precondiciones o dimensiones estructurales:

[...] la unificación de una pluralidad de demandas en una cadena equivalencial; la constitución de una frontera interna que divide la sociedad en dos campos; la consolidación de la cadena equivalencial mediante la construcción de una identidad popular que es cualitativamente algo más que la simple suma de lazos equivalenciales (Laclau 2005: 102)

Si atendemos a la primera dimensión, podríamos acordar en que el propio grito de "iNi Una Menos!" posibilitó la articulación de múltiples demandas en Argentina, desde quienes sostienen que "no hay Num sin tierra, trabajo, salud, educación y vivienda" hasta quienes reclaman "Ni Un Pibe Menos por la Droga". Pero el proceso de articulación de las demandas feministas, tal y como lo vienen reflejando las movilizaciones anuales del 8M, el Paro Internacional de Mujeres, o los Encuentros Nacionales de Mujeres, claramente excede ese grito. Los reclamos feministas tienen más de un siglo de historia en Argentina y la heterogeneidad de sus articulaciones es parte constitutiva de esa historia. Lo que sí es novedoso es

13 Tomado del Llamamiento al Paro Internacional de Mujeres (disponible en: https:// www.pagina12.com.ar/23404-el-primero-de-los-dias-por-venir. Énfasis añadido). 
el contexto social de inscripción de esas demandas, notablemente más sensible a su visibilización y escucha, conmovido por la aberración de los femicidios como el caso extremo de la violencia patriarcal, aunque esa lectura del contexto tampoco es fortuita, es fruto de la laboriosa articulación de demandas de las feministas como "demandas en contra de la violencia hacia las mujeres", demandas "en contra de la violencia patriarcal". Por eso proponemos, como un primer intento de comprender la coyuntura, que la unificación de demandas logra su equivalencia a partir de una base negativa en contra del "sistema patriarcal". Porque tal y como señala Laclau, la articulación de demandas se produce gracias a una frontera interna que divide a la sociedad en dos. Son dos aspectos de una misma condición: las demandas se reagrupan sobre una "base negativa", en relación con un poder del que se distinguen y prestan oposición (Laclau 2009: 56-7). No hay rasgos positivos, subyacentes a las demandas, que posibiliten su articulación. En otras palabras, no es necesaria la definición de "intereses estratégicos" para actuar en común: "La equivalencia procede enteramente de la oposición al poder que está más allá de la frontera" (Laclau 2009: 59), en nuestro caso, del patriarcado.

Ahora bien, si pasamos al tercer rasgo de la articulación populista, el referente a la representación y a la emergencia del sujeto popular, la continuidad de este enfoque para comprender las articulaciones de los feminismos actuales se dificulta. En un primer momento, nos mantenemos casi en el mismo lugar del análisis dado que, para Laclau, la representación del momento equivalencial comienza con la "totalización [mediante la significación] del poder que se opone al conjunto de las demandas" (Laclau 2009: 58-9) que, en nuestro caso, ya hemos identificado con el nombre de patriarcado. Pero el momento representativo no termina allí; prosigue a partir de un proceso de universalización que se hace posible cuando una demanda particular, sin abandonar su propia particularidad, "comienza a funcionar además como un significante que representa la cadena como totalidad" (Laclau, 2009:59). Ése es el momento hegemónico por excelencia, el que hace posible a la política y al populismo. Y aquí nos topamos con una primera dificultad o, mejor dicho, con dos. Para Laclau, 
[...] las condiciones de posibilidad de lo político y las condiciones de posibilidad del populismo son las mismas: ambos presuponen la división social; en ambos hallamos un demos ambiguo que es, por un lado, un sector dentro de la comunidad (los desposeídos), y, por el otro, un actor que se presenta a sí mismo, de modo antagónico, como la totalidad de la comunidad (Laclau 2009: 69). Énfasis en el original.

La primera dificultad de este planteamiento es que se equipara la política con el populismo. Mucho se ha discutido sobre las implicancias de este enfoque; ${ }^{14}$ aquí sólo señalaré que, si aceptamos esa equiparación como una de las formulaciones centrales en la obra de Laclau -esto es, que el populismo no se adscribe a un fenómeno delimitable, sino que responde "a una lógica social cuyos efectos atraviesan una variedad de fenómenos" (Laclau 2005) - el populismo deja de ser una manera posible de construir lo político para ser la única manera de hacerlo. ${ }^{15}$ La siguiente dificultad con este planteamiento es que, aun cuando podamos advertir en las movilizaciones colectivas ciertos procesos por los que un significante procura representar una cadena de demandas en su totalidad, ello no es necesariamente equivalente a reconocer que un sector de la comunidad se presenta a sí mismo como la totalidad de esa comunidad.

Procurando sortear la primera de las dificultades, Gerardo Aboy Carlés y Sebastián Barros (2013) proponen distinguir entre "lo popular" y "lo populista", otorgándonos otra manera de abordar los procesos sociales

14 Véanse Oliver Marchart 2006; Gerardo Aboy Carlés, Sebastián Barros, Julián Melo 2013.

15 En este sentido Laclau afirma que: "la pregunta si un movimiento es o no es populista" es una pregunta errónea. Lo que se debiera preguntar es: chasta qué punto es populista un movimiento? Que sería una pregunta idéntica a: chasta qué punto la lógica de la equivalencia domina su discurso? (Los dos extremos del continuum son: el discurso institucionalista dominado por una lógica de la pura diferencia y un discurso populista dominado por el de la equivalencia; en el primer caso no habría política) (68). Luego prosigue: "Si el populismo consiste en la postulación de una alternativa radical dentro del espacio comunitario, una elección en la encrucijada de la cual depende el futuro de una determinada sociedad, ino se convierte el populismo en sinónimo de política? La respuesta sólo puede ser afirmativa. Populismo significa cuestionar el orden institucional mediante la construcción de un pueblo como agente histórico —es decir, un agente que es otro en relación al orden vigente--" (Laclau 2009: 68-9). 
por los que ciertos reclamos identifican -en ciertos casos, de forma masiva- aunque esa identificación no produzca, necesariamente, una articulación populista. Como un primer desplazamiento en torno a la obra de Laclau, Aboy Carlés propone hacer una primera definición de las identidades populares como:

[...] aquel tipo de solidaridad política que emerge a partir de cierto proceso de articulación y homogeneización relativa de sectores que, plantéandose como negativamente privilegiados en alguna dimensión de la vida comunitaria, constituyen un campo identitario común que se escinde del acatamiento sin más y la naturalización de un orden vigente (Aboy Carlés 2013: 21).

Es importante advertir que, para nuestro análisis, "lo popular" no apela a sectores sociales definidos - lo que para Aboy Carlés no es más que un prejuicio sociológico, sino que se trata de una "gramática de construcción identitaria" (Aboy Carlés 2013: 21). Su definición tampoco presupone que los sectores involucrados sean mayoritarios, ni que padezcan "objetivamente" un daño social, sino solamente que así lo perciban y que esa percepción los distancie y desidentifique del poder que, según su parecer, lo provocó. Esta oposición es la que habilita la articulación de solidaridades políticas varias. Ahora bien, en el afán de reconocer lo que distingue al populismo, Aboy Carlés perfila tres formas en las que las identidades políticas populares pueden llegar a constituirse: como identidades totales, identidades parciales o identidades con pretensión hegemónica. ${ }^{16}$ Estas últimas, cuyo perfil es el que aparenta estar más próximo a las movilizaciones del num, son las que plantean sus demandas en términos universalistas y cuyas fronteras identitarias se encuentran en continuo desplazamiento. La amplia porosidad de sus fronteras es la que habilita la integración de sus adversarixs en la articulación de demandas, así como

16 Sintetizando los aspectos definitorios de las dos primeras modulaciones, las identidades totales son las que expulsan de la comunidad al campo adversario, sin posibilidad alguna de convivir con las diferencias; y las parciales son las que no aspiran a representar la totalidad comunitaria, sino más bien tienden a sostenerse como diferencias segregadas (Aboy Carlés 2013: 24-33). 
la reconfiguración de la propia identidad como efecto de esos mismos procesos articulatorios. Emergente de este marco, la identidad populista sería la que plantea una manera específica de gestionar la representación de la parte y el todo comunitario, un modo "pendular entre la ruptura y la integración" (Aboy Carlés 2013: 40) que pasa de la representación de la plebs, como sólo una parte de la comunidad, a la del populus, que ya no es parte sino el "conjunto de los miembros (sic) de una comunidad" (Aboy Carlés 2013: 27).

En sintonía con este autor, Sebastián Barros sostiene que la identificación popular tampoco emerge de la articulación con sectores populares, en términos demográficos o de clase, sino como sede de un conflicto social, de un momento de impasse político en el que se disputa el "quiénes pueden participar de aquello que tiene de común la comunidad" (Barros 2013: 44). Inspirado en la obra de Jacques Rancière, Barros señala que las demandas populares se sostienen en nombre de un daño y esperan ser audibles bajo el presupuesto de igualdad entre las partes: hay un daño que es infringido a una parte de la comunidad, pero cuyo efecto no la distingue, sino que, por el contrario, la habilita a reclamar ser parte de esa comunidad. Y al hacerlo, se produce una doble transformación: de quienes se levantan y subjetivizan en nombre del daño; y de la propia comunidad, que se conmociona en la escucha de ese grito. De allí deviene la especificidad de la identificación popular: de sus efectos políticos. Para Barros, quien en este punto sigue la propuesta de Ernesto Laclau, la emergencia de lo popular divide el campo de representación en dos: entre quienes ocupan lugares a los que legítimamente no tienen derecho y quienes rechazan dicha ocupación. Pero, a diferencia de Laclau, sostiene que de esa partición no se desprende necesariamente una articulación populista. La partición comunitaria, como efecto de la instalación de esa frontera interna en la comunidad, produce una dislocación que multiplica la emergencia de demandas sociales y, sobre todo, sus posibilidades de articulación entre sí. Si esas demandas devienen en la emergencia de "un pueblo" o en cualquier otra cosa, dependerá de la relativa estructuralidad desde la que emergen; es decir, dependerá de las condiciones materiales, así como de 
los lenguajes y narrativas disponibles. No es una consecuencia directa de la partición comunitaria. Lo distintivo del populismo es que tras esa división se erige "una nueva representación de la comunidad legítima" que opera de un modo particular sobre la relación entre las partes y el todo, sobre la base de la partición polarizada de la comunidad (Barros 2013: 54). La articulación populista entre demandas populares se produce por medio de una nueva representación comunitaria que pone un nombre al carácter excluyente de ese orden y, de ese modo, crea retroactivamente a la nueva comunidad legítima.

Si volvemos a nuestro análisis del Num, se puede reconocer cómo el mismo grito: "iNi Una Menos!" es un grito que interpela no sólo a "las víctimas" que "objetivamente" padecen la violencia machista, sino que es un grito que, como Aboy Carlés y Barros señalan, define a las demandas e identidades populares y nos solidariza con una verdad que duele. Duele no exclusivamente porque nos acusa de un daño irreversible -la muerte injusta y arbitraria de una parte de nuestra comunidad-sino, sobre todo, porque al admitirla se hace inadmisible la comunidad tal y como la vivimos. El grito "iNi Una Menos!" aclama que, aun siendo dañadxs, resistimos a la naturalización del orden vigente que nos oprime, "el patriarcado"; y al mismo tiempo, el clamor de "iVivas nos queremos!" habilita una posibilidad, o mejor aún, instiga la necesidad de una comunidad renovada. Una comunidad que no sólo incluye a lxs víctimas de la violencia machista, ni siquiera sólo a lxs feministas. Más bien apunta a mantenerse insistentemente abierta a otros daños y reclamos. Pero, ise dirige este proceso al ejercicio de una nueva representación de la comunidad?

Para responder a la pregunta que precede, es necesario abordar la segunda de las dificultades que encontramos en el planteamiento de Laclau. Es decir, el referente a las implicaciones de comprender el momento hegemónico como el de una particularidad que asume la representación de la cadena de equivalencias como una totalidad, o como aquella que se presenta, siendo la única totalidad legítima de la comunidad. En ambos casos, una parte se identifica hegemónicamente con el todo. El problema deviene no sólo en asimilar esos todos (no es lo mismo la operación de totalizar 
una cadena de equivalencias que totalizar a una comunidad), sino también en la lógica de unicidad que los presume (esperar que la heterogeneidad devenga en unidad). Como ha sido señalado por Laclau reiteradamente a lo largo de su obra, en la base de la articulación hegemónica se asienta un significante tendencialmente vacío que asume la representación universal. Cuánto más extensa sea la cadena equivalencial de demandas, más vacío de sentidos definidos deberá estar ese significante. De hecho, para Laclau, es ese vaciamiento o "pobreza" de los símbolos populistas el que le otorga su eficacia política, el que posibilita "la homogeneidad equivalencial a una realidad altamente heterogénea" (Laclau 2009: 60), la expresión al mínimo de su contenido particular. Si atendemos al proceso de movilización y extensión del ideario feminista en las movilizaciones y múltiples asambleas que se organizan en torno al "Ni Una Menos", podríamos llegar a acordar que la propia demanda que se erige en el grito "iNi Una Menos!", o el de "iVivas Nos Queremos!", aspira a representar una multiplicidad de demandas que se erigen en nombre de las "mujeres, lesbianas, trans y travestis". ${ }^{17}$ De hecho, en ambos casos se trata de demandas cuyos significantes son lo suficientemente vagos como para alojar en su seno una amplia diversidad de demandas particulares. Otro asunto sería, sin embargo, acordar que esa lógica de articulación de demandas implica que los colectivos de mujeres y feministas se presentan a sí mismos como la totalidad de la comunidad o, incluso, como la totalidad de los "feminismos". Las asambleas, colectivos y eventos que se erigen en nombre del "Ni Una Menos" no sólo son instancias de organización autónomas muy disímiles entre sí -que suelen agruparse a nivel de ciudades y/o provincias - sino que además son ampliamente excedidas por las lógicas de articulación y movilización que se producen en torno al Paro Internacional de Mujeres, la Campaña por el derecho al aborto legal, seguro y gratuito, o los Encuentros nacionales de mujeres, por sólo nombrar algunos de los procesos de organización y encuentros colectivos más reconocidos. Se trata de un complejo

17 Como fue consensuada la convocatoria en las asambleas del último 8M. Véase http:// www.resumenlatinoamericano.org/2018/03/09/8m-argentina-un-documento-que-repasa-la-crueldad-del-patriarcado-fotos/ 
entramado de movilización colectiva que a veces se intenta englobar bajo la categoría de "movimiento amplio de mujeres y feministas", aunque esa operación sea cada vez más dificultosa, dada la heterogeneidad creciente del movimiento, donde no sólo concurren mujeres (cis) con identidades partidarias, sindicalistas, obreras, así como tampoco "feministas" a secas, sino colectivas y agrupaciones que se reconocen como feminismos "lesbianos", "travestis", LGTTTBI, "socialistas", "anticapitalistas", "académicos", "populares", "comunitarios". .. Podemos acordar, entonces, que los feminismos generan en la actualidad una identificación popular, en términos de Sebastián Barros; incluso, también tienen pretensiones hegemónicas, desde el enfoque de Aboy Carlés. Lo que aún requiere mayor reflexión es la preeminencia de una articulación populista sostenida sobre una lógica de representación del todo, tanto significante como comunitaria; o, en otras palabras, la condición de privilegio de una lógica de unidad sobre la heterogeneidad en la que descansa todo proceso hegemónico.

Porque, ies posible pensar un proceso hegemónico que escape a la pretensión de totalidad, que no implique la institución comunitaria en términos de la definición de una frontera identitaria? Una de las características centrales en la distinción que realizan Aboy Carlés y Barros entre las identidades populares, por un lado, y las populistas, por el otro, es precisamente el tipo de tensión que se establece entre las partes y el todo. Aboy Carlés sostiene que uno de los errores principales en asimilar las identidades populares con las populistas fue "proyectar en toda articulación de solidaridades populares la matriz jacobina de construcción de un pueblo" (Aboy Carlés 2013: 31). Por eso distingue las identidades populistas como "un tipo particular" de las identidades con pretensión hegemónica, identidades que, aun pretendiendo cubrir al conjunto comunitario, no llegan a hacerlo del todo; sino que están siempre yendo y viniendo en un proceso de "asimilación mediante desplazamientos moleculares que suponen tanto la negociación de su propia identidad como la conversión de los adversarios a la nueva fe" (Aboy Carlés 2013: 34). A diferencia de las identidades populistas, que tienden a la polarización con sus adversarios y, en ese sentido, son "movimientos de fuerte homogeneización política" 
(Aboy Carlés 2013: 40), las fronteras identitarias de las identidades con pretensión hegemónica son porosas, tomando "la forma de manchas, con variados espacios de superposición con otras identidades adversarias" (Aboy Carlés 2013: 36). En este mismo sentido se dirige la lectura de Sebastián Barros, quien advierte que "la tensión entre parte y todo no puede caracterizar al populismo", porque esa tensión es propia de todo discurso político (Barros 2013: 57), pues existen modos específicos en los que esa tensión se resuelve en la articulación populista. Esta modalidad específica implica una nueva representación de la comunidad que, además de operar entre la parte y el todo, se orienta sobre su partición polarizada, totalizándola. Es decir, la articulación populista le otorga un nombre a la multiplicidad de identificaciones populares y, de ese modo, reclama "para sí la representación del todo comunitario", "apropiándose del todo" (Barros 2013: 57).

Si retomamos nuestra indagación acerca de un eventual devenir populista de los feminismos en las movilizaciones del \#NiUnaMenos, es difícil reconocer en el seno de sus articulaciones una pretensión totalizante. Aunque se podría reconocer en la multiplicación de "feminismos" (populares, comunitarios, sindicales, estudiantiles, etc.), la operación de nominación propia del populismo, otorgando un nuevo nombre a la representación comunitaria, esa operación dista de ser totalizadora. La extensión del feminismo como nombre parece ser, al mismo tiempo, la multiplicación de "feminismos" (populares, comunitarios, sindicales, liberales, transfeminismos...), como un proceso de mutua imbricación y contaminación de sentidos, de acuerdo con Aboy Carlés quien reconoce a las identidades con pretensión hegemónica: un proceso que, aun cuando en ocasiones pueda configurar una pintura uniforme - pensemos en la marea de pañuelos verdes frente al Congreso apoyando la legalización del aborto-, su condición más estable es la de manchas identitarias entre identidades adversarias superpuestas (pensemos en las alianzas que dieron la media sanción al proyecto de legalización del aborto en la Cámara de Diputadxs). En este sentido, las líneas de indagación que quizás debamos ampliar son las que nos posibiliten comprender los procesos hegemónicos, no tanto 
desde su dimensión de cierre, de delimitación de fronteras identitarias bajo la pretensión de totalizar la plenitud ausente de la comunidad (como la articulación populista), sino desde su configuración equivalencial, lo que el propio Laclau reconoció como distintivo de la configuración mítica en Sorel; es decir, desde los efectos de movilización y multiplicación de luchas que se unifican —de maneras más o menos estables—en su nombre. ${ }^{18}$

18 Recordemos que Laclau analiza la concepción del mito en la obra de Georges Sorel como un modo de confirmar la configuración equivalencial en todo proceso ideológico, y se detiene en lo que considera las tres contraposiciones básicas de su pensamiento: fuerza/violencia, utopía/mito, huelga política general/huelga proletaria general. En cada caso, establece que lo importante es notar cómo el segundo de los términos difiere del primero "en razón del carácter equivalencial que sus componentes establecen entre sí" (Laclau 2002: 48). Así, a diferencia de la utopía como "construcción intelectual [...] de una sociedad enteramente realizada", el mito "es un conjunto de imágenes equivalentes, capaces de galvanizar el imaginario de las masas y lanzarlas a la acción colectiva" (Laclau 2002: 49). Ello se debe a que "los contenidos particulares del mito son sustituibles el uno por el otro [y es por esto que deben ser aprehendidos como conjunto] en la medida en que todos ellos simbolizan una plenitud ausente, y su eficacia debe ser medida por la movilización que se deriva de sus efectos equivalenciales, no por el éxito de sus contenidos literales diferenciados" (2002: 50). Es en este sentido que considera a la huelga proletaria como un mito: "ella no es un evento real separado de las huelgas políticas reales, sino una dimensión que unifica, de modo equivalencial, a una variedad de luchas y acciones durante un largo periodo histórico. Que un hecho concreto pertenezca a la acción política o a la acción proletaria es algo que es, en última instancia, indecidible, y que está siempre abierto a una pluralidad de lecturas e intervenciones estratégico-discursivas [...] mientras en el caso de la huelga política la lucha se agota enteramente cuando su objetivo ha sido alcanzado, en la huelga proletaria cada enfrentamiento parcial es el pretexto para mantener vivo y entrenar al proletariado como agente revolucionario. En el caso del segundo término de cada una de las distinciones, lo particular es el medio de representación de algo que lo trasciende" (2002: 52). En nuestro caso, la dimensión mítica del feminismo es lo que posibilita que sus luchas disímiles se presenten de un modo equivalente ("No hay Ni Una Menos sin Aborto Legal", "No hay Ni Una Menos sin techo, salud, vivienda", etc.), y lo que mantiene siempre movilizados a sus sectores. Siempre hay un "horizonte feminista" al que llegar, más allá de los éxitos parciales que se vayan alcanzando. Y cada vez son más los hechos que se leen como parte de la "revolución" en ciernes (Peker 2017). Una lectura que no sólo apunta a un "porvenir" feminista, sino que revisa su propio pasado. Para una lectura en esta línea véanse Macón, Solana 2015. 
Natalia MartíneZ

EL (DES)ORDEN DEL FEMINISMO

(...) afirmamos el derecho a decir NO frente aquello que no se desea: una pareja, un embarazo, un acto sexual, un modo de vida preestablecido. Afirmamos el derecho a decir No a los mandatos sociales de sumisión y obediencia.

\# Ni Una Menos. Del Documento leído en la Plaza de los Dos Congresos, 3/06/2015

Uno de los sentidos más insistentes de las movilizaciones que emergieron tras el grito de "Ni Una Menos" es el de la negación, el rechazo: el sentido del "Ni" que se actualiza en la historia de los feminismos latinoamericanos al de " $n i$ una más" mexicano, que ahora llega a reformularse en las calles por un extendido: " $n i$ por estudiante, $n i$ por mapuche, $n i$ por mujer", o el propio reclamo del epígrafe sobre "el derecho a decir no frente aquello que no se desea", son algunas de las maneras que circularon y circulan en las convocatorias del num y que cada vez que se pronuncian, se gritan, promulgan un rechazo, marcan un límite. Este límite no se señala desde un afuera invulnerable, desafectado por los vínculos opresivos que denuncia; emerge desde las propias garras del poder que asesina y de la mano de una tradición política singular: el feminismo. Es un límite que, asumiendo lo personal de lo político y lo político de lo personal, se organiza en colectivo y se grita en la intimidad singular de su modus operandi.

El enfoque populista de la política, en los términos generales planteados en la obra de Ernesto Laclau, es muy insistente en señalar cómo toda demanda aparece internamente dividida: por un lado, desde su contenido particular, su especificidad; por el otro, desde el rechazo al poder contra el cual esa demanda se formula. Las demandas pueden ser muy diferentes y distanciadas entre sí, pero es su común rechazo a un poder al que se oponen desde donde todas son equivalentes. Propongo en este apartado partir desde este aspecto, el del común rechazo de las demandas, aunque no para corroborar la preeminencia de una eventual lógica articulatoria populista, sino para comprender esas articulaciones desde sus efectos de subjetivación, efectos que se explicitan en la propia formu- 
lación singular de un "no", "así no", "ya no". Lo que propongo es atender a la negatividad del reclamo, no en términos de demanda insatisfecha, negatividad concebida como falta o plenitud ausente de la comunidad, sino atendiendo a la negación como práctica política, como una afirmación sobre lo que no se desea. Desde esta perspectiva, atenderíamos el rechazo como una lógica que, no sólo eventualmente unifica, o que puede llegar a hacerlo, sino como aquella que inaugura un proceso de des-sujeción, de desidentificación -en términos rancerianos- con el potencial de alterar el reparto del orden de lo sensible y las condiciones de posibilidad del ser.

Esta recuperación de la obra de Rancière nos posibilita comprender las movilizaciones del NuM como esa irrupción de lxs que no tienen parte y que, de ese modo, desordenan el orden policial, "natural", que les había sido asignado. Quienes irrumpen - en nuestro caso, la irrupción se hizo más mediáticamente visible en nombre del daño ejercido sobre las mujeres cis, heterosexuales- provocan una distorsión en el orden instituido y, de esa forma, habilitan nuevos modos de subjetivación política. Para Rancière, esta subjetivación se hace posible previa desidentificación -como "arrancamiento a la naturalidad de un lugar"- y "produce una multiplicidad que no estaba dada en la constitución policial de la comunidad, una multiplicidad cuya cuenta se postula como contradictoria con la lógica policial" (Rancière 2007: 53). Una de las referencias más comunes de esa multiplicidad, a la que alude el autor francés, es el propio "pueblo". Aunque aquí hay una gran diferencia con la noción de "pueblo" - y la lógica populista-de Ernesto Laclau. El nombre del "pueblo" en Rancière, antes que una convocatoria a la unidad equivalencial de sus diferencias, insta a la desunión de la comunidad consigo misma. "El pueblo, al mismo tiempo, siempre es más o menos que sí mismo" porque ofrece su nombre a cualquiera que requiera la inscripción de su daño, un daño ejercido por la misma comunidad a quien se lo grita (Rancière 2007: 24). ${ }^{19}$ En nuestro análisis, el proceso que viene habilitando la multiplicación de los reclamos bajo el num hace cada vez más audibles y visibles no sólo los reclamos de las

19 Para un análisis de otras diferencias en la noción de pueblo en Laclau y Rancière, véase Federico Galende 2016. 
mujeres cis heterosexuales, sino también los que se producen en nombre del colectivo trans, lesbianas, estudiantes, trabajadorxs precarizadxs, trabajadorxs sexuales, desaparecidxs por trata, mapuches... Por ello, quizás, se pueda entender como un proceso de subjetivación política a la ranceriana, incluso uno que aluda, desde esta perspectiva, a la configuración de un pueblo.

La dificultad deviene, sin embargo, si desde esta perspectiva procuramos analizar las implicaciones o efectos políticos de esa misma subjetivación, así como sus límites. Es decir, si movemos el foco de nuestra atención desde el momento dislocatorio de la distorsión (tort), a la potencia subjetiva que se inaugura. Porque incluso cuando la fuerza distorsiva se sostenga en cada encuentro o movilización del NuM, habilitando el grito de nuevos daños (tort) y desidentificaciones, aun cuando la gramática y la práctica de la negación mantenga su potencia de transformación, en tanto desobediencia y desapego de las relaciones de poder que imperan, resta reconocer los horizontes políticos que cada una de esas subjetivaciones políticas abren (o cierran). En otras palabras, además de la multiplicidad de diferencias que la desidentificación anuncia, quedan por reconocer los universales por cuya proclamación se promete un nuevo ordenamiento policial, más justo. Y aquí no me refiero al gesto que, en palabras de Zizek, "se activa cuando una demanda particular representa el gesto universal de rechazar el poder que sea" (Zizek 2016: 263) ${ }^{20}$ Gesto que podría sintetizarse en la proclama: "Si nos tocan a una, nos tocan a todas" o, mejor aún, en la que advierte: "Nos organizamos para cambiarlo todo". ${ }^{21}$ Me refiero más bien a reconocer cuál es el potencial que se abre desde cada una de las subjetivaciones políticas emergentes en la configuración de un nuevo orden policial de los cuerpos; es decir, su potencialidad para habilitar otros lugares y modalidades donde ubicarnos, otros modos del hacer, del ser y del decir. Porque la gestualidad del rechazo a que "nos toquen" no

20 En este sentido, para Rancière habría un solo universal en la política, un "universal polémico", que es la igualdad que se asume en la figura de la distorsión. Rancière 2007: 56.

21 Proclama establecida en la convocatoria en Argentina al Paro Internacional de Mujeres el 8 de marzo del 2017. Véase https://www.pagina12.com.ar/23404-el-primero-delos-dias-por-venir 
implica lo mismo para "todxs", como tampoco la organización colectiva apunta a transformar lo mismo. Se hace necesario analizar, en cada caso, las superficies de inscripción desde donde "todxs" nos movilizamos y organizamos, así como los horizontes singulares de transformación al que apuntamos. Y para abordar este problema, la formulación que Rancière hiciera sobre el pueblo -ligada a la subversión del orden instituido, más que a su configuración- quizás no sea la más adecuada. Si quisiéramos proseguir con Rancière, la noción que se corresponde mejor a las preguntas que quisiera abordar es la de "policía". Recordemos que esta categoría se refiere a una lógica que ordena los cuerpos en el espacio, definiendo

[...] las divisiones entre los modos del hacer, los modos del ser y los modos del decir, que hace que tales cuerpos sean asignados por su nombre a tal lugar y a tal tarea; es un orden de lo visible y lo decible que hace que tal actividad sea visible y que tal otra no lo sea, que tal palabra sea entendida como perteneciente al discurso y tal otra al ruido (Rancière 2007: 45).

Desde esta perspectiva, ¿cuáles serían los ordenamientos de los cuerpos que se ponen a disposición en las movilizaciones del Num? ¿qué actividades se visibilizan?, ¿qué voces se escuchan?, ¿qué otros lugares se ocupan? Aunque aún no es el momento para responder a estas preguntas, que apenas ahora es posible formular, espero con este artículo posibilitar un primer paso desde el cual comenzar.

REFLEXIONES FINALES: FEMINISMO:

¿QUÉ NOS DICE EL NOMBRE?

De una manera sorprendente $-\mathrm{y}$ emocionante para quienes nos inscribimos en esta corriente- el feminismo se hizo masivo. Es como si ya no fuera posible detener el flujo de sus olas, que desde hace casi tres siglos se vienen produciendo, y, de hecho, estuviésemos en medio de una marea feminista. Tal y como señalaba una periodista recientemente, tratando de comprender este fenómeno: 
[...] hasta hace apenas unos años, ser feminista era efectivamente una militancia y, para el lugar común, la idea o la representación de una mujer feminista recorría el arco que iba desde la demente que odiaba a los hombres y gritaba consignas contra el patriarcado hasta descalificaciones y groserías de todo orden vinculadas a sus supuestas elecciones sexuales. Mamá no podía ser feminista; la abuela tampoco. Las fotos de estos días nos deja a todos en claro que esa condición mutó (Pomeraniec 2018).

Sí, está claro, la representación del feminismo cambió. Ya no sólo representa a fanáticas y lesbianas, hoy también representa a madres y abuelas, trabajadoras sindicales y amas de casa; también a homosexuales, travestis y padres de familia. ¿Significa esto que hay un pueblo feminista? Como he tratado de señalar a lo largo de este artículo, nuestra respuesta dependerá de lo que concibamos como propio y definitorio de ese pueblo. En principio, considero que esta posibilidad es plausible sólo si descartamos, como una condición instituyente y necesaria, la pretensión de mitigar su heterogeneidad. El feminismo es hoy una identidad popular, o más precisamente, posibilita una identificación popular que excede la singularidad de sus reclamos y se universaliza; es decir, tiene pretensiones hegemónicas. No representa una demanda específica, como pueden llegar a ser el derecho al aborto legal, seguro y gratuito o una vida sin violencia. Es una identificación que continuamente está añadiendo demandas diferentes, que se encadenan a un sentido cada vez más amplio que se inscribe en su nombre. Por eso "feministxs", hoy, podemos ser todxs.

Ahora bien, ¿qué sentido tiene hoy nombrarse como "feminista"? Es decir, ¿qué sentidos se instalan al nombrarnos como feministas y cuáles se excluyen? Porque, que todxs podamos identificarnos como feministas, no implica que signifique lo mismo para todxs. Como también he procurado exponer en este artículo, abordar estas preguntas requiere, en primer lugar, relegar las perspectivas que tienden a privilegiar una epistemología feminista, es decir, aquellas que conciben un modo preestablecido de definir los "intereses estratégicos de género". Estas preguntas sólo se pueden responder de un modo situado, a partir de un análisis de las narrativas que circulan y desde donde se inscribe un nombre como el de "feminista". 
Sólo desde una aproximación semejante podremos delimitar esos lenguajes y reconocer sus fronteras, saber qué está diciendo quien se nombra como "feminista".

En segundo lugar, reconocer estos sentidos también requerirá un desplazamiento de las lógicas totalizadoras que interceden en la construcción política de un pueblo, al menos desde una perspectiva que concibe la construcción del campo popular íntimamente vinculada a la lógica populista. La potencia analítica de semejante enfoque radica en atender la articulación de los feminismos por la delimitación de sus fronteras, por lo que rechaza, y no por los intereses estratégicos que eventualmente defina. En este sentido, es de suma importancia reconocer lo que el feminismo combate, lo que apunta como un límite, de lo que aspira a des-identificarse; aunque eso no alcance para comprender lo que significa. Que "el patriarcado" y sus relaciones de opresión sean lo que marca las fronteras del feminismo, como una fuerza antagonista, no es suficiente para identificar los sentidos que el feminismo abraza. Es un comienzo ineludible; de hecho, es lo que habilita la emergencia del feminismo, lo que les da sentido y fuerza a sus demandas y articulaciones. Pero no es lo que define los horizontes de lo que su nombre aclama. Reconocer estos horizontes nos obliga a atender también los procesos de subjetivación política que la desidentificación habilita, el nuevo ordenamiento de los cuerpos para hacer, ser y decir. Y éste es un proceso apenas emergente. Comprender el devenir de todas estas dimensiones -siempre incierto- es la tarea que nos aguarda para quienes estamos comprometidxs en un porvenir feminista.

BIBLIOGRAFÍA

Di MARCO, GRACIELA. El pueblo feminista. Movimientos sociales y lucha de las mujeres en torno a la ciudadanía. Buenos Aires: Biblos, 2011.

GALENDE, FEDERICO. "Rancière, arte, performance y teoría". Andrés Maximiliano Tello (ed.). Gobierno y desacuerdo. Diálogos interrumpi- 
dos entre Foucault y Rancière. Viña del Mar: Asociación Communes, 2016: 173-194.

Guzmán, Virginia, Patricia Portocarrero, Virginia Vargas. Una nueva lectura: género en el desarrollo. Lima: Entre Mujeres, 1991.

Jelin, Elizabeth, "Ciudadanía e identidad. Una reflexión final". Jelin (comp.). Ciudadanía e Identidad: Las mujeres en los movimientos sociales latino-americanos. Ginebra: UNSRID. 1987. 311-351.

LACLAU, ERNESTO. Misticismo, retórica y política. Buenos Aires: FCE, [2002] 2006.

La razón populista. Buenos Aires: FCE, [2005] 2007.

."Populismo: ¿Qué nos dice el nombre propio?". Francisco Panizza (comp.). El populismo como espejo de la democracia. Buenos Aires: FCE, 2009. 51-70.

LÓPEZ, MARíA PÍA. "Ni una menos: aquelarre y algarabía". Página 12, Sección Sociedad, 2 de junio, 2015. Artículo en línea disponible en https://www.pagina12.com.ar/diario/sociedad/3-273992-2015-06-02. html (Consultado el 2 de junio de 2015).

. "Duelo colectivo y templanza de los cuerpos". Anfibia, s/f. Artículo en línea disponible en http://www.revistaanfibia.com/cronica/ duelo-colectivo-y-templanza-de-los-cuerpos/

Macón, Cecilia; Mariela Solana (eds.). Pretérito indefinido. Afectos y emociones en las aproximaciones al pasado. Buenos Aires: Recursos Editoriales, 2015.

MARCHART, OlIVER. "En el nombre del pueblo. La razón populista y el sujeto de lo político". Cuadernos del CENDES 23. 62 (mayo-agosto 2006): 37-58.

MartíneZ, Natalia, JuAn Manuel Reynares. "Devenir pueblo: la eventualidad hegemónica del Estado en la obra de Laclau”. Utopía y Praxis Latinoamericana. 19. 66 (julio-septiembre 2014): 109-120.

MarTínEZ, NATALIA "Sobre los límites (políticos) de una epistemología feminista". Sapere Aude 5.9 (2014): 287-305. 
"La emergencia del feminismo en la Argentina. Un análisis de las tramas discursivas a principios de siglo". Estudos Feministas 23. 1 (2015): 71-97.

MolineauX, MaXine. "Mobilization without Emancipation? Women's lnterests, the State, and Revolution in Nicaragua". Feminist Studies 11. 2 (1985): 227-254.

. "Analysing Women's Movements". Development and Change 29 (1998): 219-245.

- Movimientos de mujeres en América Latina. Estudio teórico comparado. Madrid: Ediciones Cátedra/Instituto de la mujer/ Universidad de Valencia, 2003.

Moser, CAROLInE. Planificación de género y desarrollo. Teoría, práctica y capacitación. Lima: Flora Tristán Ediciones, 1995.

Ni una MENos. Sitio Web Oficial. "Documento leído el 3 de junio 2015", Sección "En qué creemos y qué pedimos". http://s1000050.ferozo. com/wp-content/uploads/2015/06/documento_OK-1-1.pdf (Consultado el 4 de junio de 2015).

PANZERINI, LoRena. "Un crimen espeluznante". Artículo en línea disponible en https://www.pagina12.com.ar/61678-un-crimen-espeluznante (Consultado el 10 de noviembre de 2016).

PEKER, LuCIANA. La revolución de las mujeres. No era sólo una píldora. Villa María: Eduvim, 2017.

Pomeraniec, Hinde. "La verdadera revolución son ellas". Infobae, 8 de marzo, 2018. Artículo en línea disponible en https:/www.infobae.com/ opinion/2018/03/08/a-verdadera-revolucion-son-ellas/ (Consultado el 9 de marzo de 2018).

Rancière, JaCQues. El desacuerdo. Política y filosofía. Buenos Aires: Nueva Visión, 2007.

ZERILLI, LINDA. El feminismo y el abismo de la libertad. Buenos Aires: FCE, 2008.

ZizeK, SlaVoj. "La lección de Rancière". Andrés Maximiliano Tello (ed.). Gobierno y desacuerdo. Diálogos interrumpidos entre Foucault y Rancière. Viña del Mar: Asociación Communes, 2016: 249-268. 